\title{
Dysbindin-1 is reduced in intrinsic, glutamatergic terminals of the hippocampal formation in schizophrenia
}

\author{
Konrad Talbot, ${ }^{1}$ Wess L. Eidem, ${ }^{1}$ Caroline L. Tinsley, ${ }^{2}$ Matthew A. Benson, ${ }^{2}$ Edward W. Thompson, ${ }^{1}$ \\ Rachel J. Smith, ${ }^{3}$ Chang-Gyu Hahn, ${ }^{1}$ Steven J. Siegel,, John Q. Trojanowski, ${ }^{4}$ Raquel E. Gur, ${ }^{1}$ \\ Derek J. Blake, ${ }^{2}$ and Steven E. Arnold ${ }^{1}$ \\ 1Department of Psychiatry (Center for Neurobiology and Behavior), University of Pennsylvania School of Medicine, Philadelphia, Pennsylvania, USA. \\ 2Department of Pharmacology, University of Oxford, Oxford, United Kingdom. ${ }^{3}$ Department of Neuroscience and ${ }^{4}$ Department of Pathology \\ and Laboratory Medicine, University of Pennsylvania School of Medicine, Philadelphia, Pennsylvania, USA.
}

\begin{abstract}
Eleven studies now report significant associations between schizophrenia and certain haplotypes of singlenucleotide polymorphisms in the gene encoding dysbindin-1 at $6 \mathrm{p} 22.3$. Dysbindin-1 is best known as dystrobrevin-binding protein 1 (DTNBP1) and may thus be associated with the dystrophin glycoprotein complex found at certain postsynaptic sites in the brain. Contrary to expectations, however, we found that when compared to matched, nonpsychiatric controls, $73-93 \%$ of cases in two schizophrenia populations displayed presynaptic dysbindin-1 reductions averaging $18-42 \%(P=0.027-0.0001)$ at hippocampal formation sites lacking neuronal dystrobrevin (i.e., $\beta$-dystrobrevin). The reductions, which were not observed in the anterior cingulate of the same schizophrenia cases, occurred specifically in terminal fields of intrinsic, glutamatergic afferents of the subiculum, the hippocampus proper, and especially the inner molecular layer of the dentate gyrus (DGiml). An inversely correlated increase in vesicular glutamate transporter-1 (VGluT-1) occurred in DGiml of the same schizophrenia cases. Those changes occurred without evidence of axon terminal loss or neuroleptic effects on dysbindin-1 or VGluT-1. Our findings indicate that presynaptic dysbindin-1 reductions independent of the dystrophin glycoprotein complex are frequent in schizophrenia and are related to glutamatergic alterations in intrinsic hippocampal formation connections. Such changes may contribute to the cognitive deficits common in schizophrenia.
\end{abstract}

\section{Introduction}

Genetic susceptibility plays an important role in the pathogenesis of schizophrenia, the heritability of which is now estimated to be $82-84 \%$ (1). Since schizophrenia is a severe mental disorder with a lifetime prevalence of $0.55 \%$ worldwide (2), the search for genetic susceptibility loci has been intensive (3-7). In 2002, significant associations were reported for the first time between schizophrenia and haplotypes of single-nucleotide polymorphisms (SNPs) in specific genes (6-9). Among those were sets of SNPs in the gene encoding dysbindin-1, situated at chromosome locus 6p22.3 (10). Other known forms of dysbindin (dysbindin-2 and dysbindin-3) are not encoded at known schizophrenia-susceptibility loci (M. Benson and D. Blake, unpublished observations).

A significant association between schizophrenia and various dysbindin-1 haplotypes has now been reported in populations from England (11), Ireland (10, 12, 13), Wales (13), The Netherlands (14), Germany/Hungary/Israel (15), Sweden (16), Bulgaria (17), China, $(18,19)$, and Japan (20). Only two studies have failed to replicate such findings. One analyzed an Irish population (21) in which sub-

Nonstandard abbreviations used: cornu ammonis fields $1-3$ of the hippocampus (CA1-3); dentate gyrus (DG); DG granule cell layer (DGg); DG hilus (DGh); DG inner molecular layer (DGiml); diaminobenzidine (DAB); hippocampal formation (i.e., CA1-3 + DG + subiculum) (HF); neuronal nucleus antibody (NeuN); postmortem interval (PMI); single-nucleotide polymorphism (SNP); vesicular glutamate transporter-1 (VGluT-1).

Conflict of interest:The authors have declared that no conflict of interest exists.

Citation for this article: J. Clin. Invest. 113:1353-1363 (2004).

doi:10.1172/JCI200420425. sequent analysis showed that a haplotype containing an SNP in the dysbindin-1 promoter region actually was more common in patients with schizophrenia than in control subjects $(6,13)$. The remaining negative study, of German and Polish populations (16), did not test haplotypes that included the promoter SNP.

The dysbindin- 1 gene is thus among the most promising susceptibility genes for schizophrenia $(6,7,22)$. While two preliminary studies on prefrontal brain homogenates report decreased dysbindin gene (23) and protein expression (24) in schizophrenia, it remains unclear how such alterations may contribute to the cognitive and behavioral features of the disorder. Our limited knowledge of the protein is nevertheless sufficient to suggest hypotheses testable with immunohistochemistry. Dysbindin-1 is a 40- to 50-kDa protein expressed neuronally (25) in many areas of the human brain (10). It is best known as a dystrobrevin-binding protein 1, DTNBP1, capable of binding both $\alpha$ - and $\beta$-dystrobrevins $(25,26)$. Dystrobrevins are components of the dystrophin glycoprotein complex (27), which is concentrated at postsynaptic densities in diverse brain areas $(28,29)$. Both $\alpha$ - and $\beta$-dystrobrevin are found in the brain $(29,30)$, but only the $\beta$ isoform is expressed in neurons, where it is associated primarily with postsynaptic densities (29).

Dysbindin-1's ability to bind $\beta$-dystrobrevin and thus interact with the dystrophin glycoprotein complex at synaptic sites implies that dysbindin-1 polymorphisms may enhance susceptibility to schizophrenia by altering synaptic mechanisms. Such alterations are among the few neurobiological hallmarks of schizophrenia $(31,32)$. Given evidence of relatively high allelic variation in regu- 


\title{
Table 1
}

\author{
Case demographics
}

\begin{tabular}{lcc} 
& \multicolumn{2}{c}{ University of Pennsylvania cases } \\
Parameters & Control $(n=17)$ & Schizophrenia $(n=17)$ \\
Mean age: $y r$ (range) & $79.3(65-93)$ & $78.4(68-90)$ \\
Sex ratio: female/male & $12 / 5$ & $12 / 5$ \\
Mean PMI: $h$ (range) & $12.1(3.5-26)$ & $13.5(7-24)$ \\
Hemisphere studied: left/right & $10 / 7$ & $10 / 7$
\end{tabular}

\begin{tabular}{cc}
\multicolumn{2}{c}{ Stanley Foundation cases } \\
Control $(n=15)$ & Schizophrenia $(n=15)$ \\
$48.1(29-68)$ & $44.2(25-62)$ \\
$6 / 9$ & $6 / 9$ \\
$23.7(8-42)$ & $33.7(12-61)$ \\
$7 / 8$ & $6 / 9$
\end{tabular}

latory sequences of the dysbindin-1 gene in the human brain (33), we tested the hypothesis that dysbindin-1 protein levels are altered at synaptic sites in multiple brain areas of individuals with schizophrenia. Priority was given to immunohistochemical tests, because they allow anatomical localization of any synaptic protein changes within specific neural systems. This is the level of brain organization at which genetic variations can affect cognition and behavior (34). We focus here on the hippocampal formation (HF), because it displays an unusual combination of features important to the present study: postsynaptic densities linked to the dystrophin glycoprotein complex $(35,36)$, frequent synaptic abnormalities in schizophrenia $(37,38)$, and an important role in mediating cognitive and behavioral aspects of the disorder (39).

\section{Methods}

Research design and subjects studied. A matched-pairs design was used to compare postmortem tissue from 17 nonpsychiatric controls and 17 patients with schizophrenia who had participated in a longitudinal study of prospectively diagnosed subjects that was approved by an Institutional Review Board at the University of Pennsylvania and conducted by the Schizophrenia Research Center at that university (40). Autopsy consent was obtained from a family member or legal guardian in all cases. Psychiatric subjects met DSM-IV (Diagnostic and Statistical Manual of Mental Disorders, fourth edition; American Psychiatric Association, Washington, DC, USA) diagnostic criteria for schizophrenia as determined in consensus conference after review of medical records, direct clinical assessments of patients, and interviews with caregivers. Control and psychiatric subjects were matched for sex, age (within 5 years), postmortem interval (PMI; within 7 hours), fixative used (ethanol, formalin, or Bouin's fixative), and cerebral hemisphere studied (left or right). Neuropathological examination revealed no degenerative processes in control or psychiatric cases (i.e., there was no evidence of gross cell loss, infarcts, or abnormal densities of amyloid plaques, neurofibrillary tangles, or Lewy bodies).

A second sample of 15 nonpsychiatric and 15 schizophrenia cases in the Stanley Foundation's standardized collection of formalinfixed brains (41) was studied. They were matched for sex and age (within 10 years); there were no significant group differences between control and schizophrenia cases in PMI or hemisphere studied. Demographic data on the University of Pennsylvania and Stanley Foundation cases are summarized in Table 1. Tissues from 15 bipolar and 15 major-depressive cases from the Stanley Foundation were also studied.

Tissue preparation. Coronal brain slabs containing intermediate rostrocaudal levels of the HF and several other brain regions (e.g., anterior cingulate cortex, superior temporal cortex, striatum, and cerebellum) were removed at autopsy. Tissue from one hemisphere was fresh-frozen at $-80^{\circ} \mathrm{C}$ for Western blotting and in situ hybridization. Tissue from the other hemisphere was fixed in neutralbuffered formalin (10\%), ethanol (70\% in saline), or Bouin's fixative for 24-48 hours and embedded in paraffin. Fixed tissue from the University of Pennsylvania sample was cut coronally at $6 \mu \mathrm{m}$ on a rotary microtome tested to assure invariant section thickness, and mounted on 3-aminopropyltriethoxysilane-coated slides (42). Tissue from the Stanley Foundation sample was cut coronally at $10 \mu \mathrm{m}$ in the case of formalin-fixed, paraffin-embedded blocks for immunohistochemistry, or $14 \mu \mathrm{m}$ in the case of fresh-frozen material for in situ hybridization.

In situ bybridization. Dysbindin-1 gene expression was studied in six normal Stanley Foundation cases (mean age 49.7 years) by in situ hybridization on sections through the HF and adjoining cortical areas.

Radiolabeled sense and antisense riboprobes were synthesized using a cDNA for human dysbindin-1 as template. The cDNA (AI337229; American Type Culture Collection, Manassas, Virginia, USA; catalog no. 3064549) encompassed 531 C-terminal nucleotides of a 1,300-nucleotide transcript of human dysbindin. It was inserted into PT7T3pac vectors. The plasmids were linearized by digestion with HindIII for synthesis of sense probes or XhoI for antisense probes. Riboprobes were then synthesized using T7 (for sense probes) or T3 (for antisense) RNA polymerase in the presence of $\alpha^{35}$ S-CTP and $\alpha^{35}$ S-UTP (Amersham Biosciences, Piscataway, New Jersey, USA). The sense and antisense probes were size-fractionated on a sequencing gel. Those probes yielding distinct bands were used for hybridization.

Frozen tissue sections were prepared for in situ hybridization by immersion in 4\% paraformaldehyde ( $\mathrm{pH} 7.4$ ) for 10 minutes and then rinsed sequentially in $0.1 \mathrm{M}$ triethylethanolamine (TEA), 0.1 M TEA plus $0.25 \%$ acetic anhydride, and $2 \times$ saline sodium citrate (SSC, pH 7.0). Probes labeled with $\alpha^{35}$ S-CTP and $\alpha^{35}$ S-UTP were added to the slides $\left(9.0 \times 10^{5} \mathrm{cpm}\right.$ per slide $)$ in $90 \mu \mathrm{l}$ of hybridization buffer (50\% formamide, $300 \mathrm{mM} \mathrm{NaCl}, 20 \mathrm{mM}$ Tris [pH 8.0], 5 mM EDTA, $1 \times$ Denhardt's solution, $10 \%$ dextran sulfate [50\% wt/ vol], $10 \mathrm{mM}$ DTT [dithiothreitol]). Slides were then covered with Parafilm (Fisher Scientific, Pittsburgh, Pennsylvania, USA) and incubated for 18 hours at $55^{\circ} \mathrm{C}$, after which they were washed in $2 \times$ SSC for 15 minutes twice. The sections were next treated with RNase A solution $(20 \mathrm{mg} / \mathrm{ml}$ in $500 \mathrm{mM} \mathrm{NaCl}$ and $10 \mathrm{mM}$ Tris $)$ for 30 minutes at $37^{\circ} \mathrm{C}$. A serial stringency washing was performed by rinsing in $2 \times \mathrm{SSC}$ at room temperature for 10 minutes twice and then in $0.1 \times \mathrm{SSC}$ at $65^{\circ} \mathrm{C}$ for 2 hours. Air-dried slides were exposed to emulsion (NTB-2; Eastman Kodak, Rochester, New York, USA) for 9 days, developed in D19 (Eastman Kodak), stabilized in fixative Eastman Kodak), and counterstained lightly with cresyl violet. Only background labeling was seen with the sense probe (Figure 1F). 


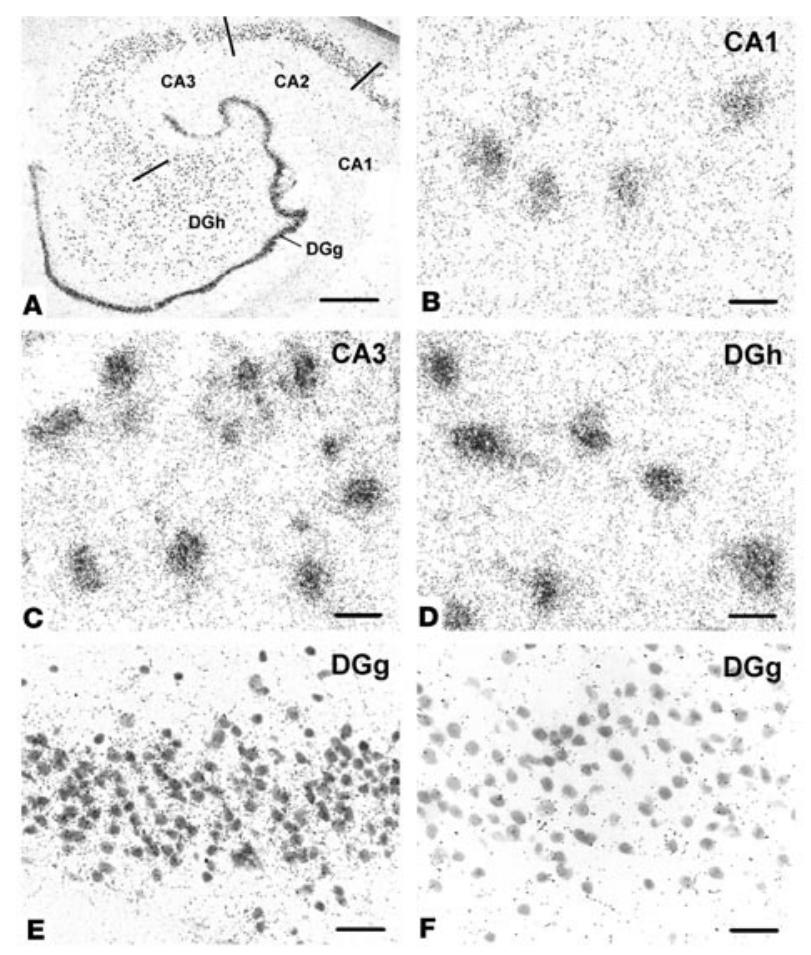

Antibodies and antibody specificity. Dysbindin-1 was detected with a rabbit polyclonal antibody (PA3111Ae2) raised against the Cterminus of mouse dysbindin (amino acids 196-352). PA3111Ae2 recognizes peptides of the same molecular weight as dysbindin isoforms in COS-7 cells only when they are transfected with a dysbindin-1 expression construct (Figure 2A). The antibody does not recognize other proteins expressed by COS-7 cells transfected with expressed sequence tags bearing significant similarity to the dysbindin-1 gene (e.g., dysbindin-2; Figure 2B). PA3111Ae2's ability to recognize antigen (Figures 3 and 4) was blocked by preadsorption with $10^{-7} \mathrm{M}$ M10FL, a recombinant, full-length dysbindin-1 peptide (Figure 4, compare C and D). Omission of PA3111Ae2 (or any other antibody used in this study) resulted in immunohistochemical runs without visible reaction product. Preliminary tests with a second dysbindin-1 antibody raised against the complete mouse protein (rabbit polyclonal antibody M10FLA) gave essentially the same pattern of immunoreactivity as PA3111Ae2 in mouse and human brain tissue.

$\beta$-Dystrobrevin was detected with a rabbit polyclonal antibody $(\beta 521)$ raised against a unique $\mathrm{C}$-terminal region of the mouse antigen (30). A neuronal nucleus antibody (NeuN) (MAB377; Chemicon International Inc., Temecula, California, USA) was used to label neurons selectively. Presynaptic terminals were identified with mouse mAb's against synaptophysin (M0776; DAKO Corp.,

\section{Figure 2}

Tests of dysbindin-1 antibody specificity in COS-7 cells. Such cells normally express no detectable dysbindin. (A) The antibody PA3111Ae2 (1:100) recognizes dysbindin-1 only in COS-7 cells transfected with dysbindin-1 expression constructs. It does not recognize antigens in COS-7 cells transfected with dysbindin-2 expression constructs. (B) The antibody M10RP-FLA (1:50) recognizes dysbindin-2, which is of lower molecular weight than dysbindin-1. (C) Equal loading of samples was demonstrated with $\beta$-tubulin antibody T4026 from Sigma-Aldrich (1:5,000).

\section{Figure 1}

Dysbindin gene expression in normal human HF, demonstrated by in situ hybridization. The tissue was counterstained for Nissl substance. All principal neurons in the DG and CA1-3 were labeled with the antisense riboprobe, as seen at low magnification (A). Gene expression in CA1 (B) and in the DGg (E) is lower than in CA3 (C) or in the DGh (D). Comparison of DGg with antisense $(E)$ and with sense riboprobes $(\mathbf{F})$ shows that the latter produced very little cell labeling. Scale bar in A: 1 $\mathrm{mm}$; scale bars in B-F: $20 \mu \mathrm{m}$.

Carpinteria, California, USA) and synapsin-1 (106 001; Synaptic Systems GmbH, Göttingen, Germany). Glutamatergic terminals common in the HF (43) were visualized selectively with a guinea pig polyclonal antibody to vesicular glutamate transporter-1 (VGluT-1) (AB5905; Chemicon International Inc.).

Western blotting. Fresh-frozen brain tissue was used from four normal human cases with low PMIs (3.5-7 hours) in the University of Pennsylvania brain bank. Western blotting was performed as described previously $(25,30)$ using dysbindin- 1 antibody PA3111Ae2 (1:100). Blots were incubated at $4^{\circ} \mathrm{C}$ overnight in the primary antibody, washed thoroughly, and incubated for 1 hour at room temperature with an HRP-conjugated anti-rabbit secondary antibody (Jackson ImmunoResearch Laboratories Inc., West Grove, Pennsylvania, USA). They were developed with a chemiluminescence detection kit (Pierce Biotechnology Inc., Rockford, Illinois, USA).

Immunohistochemistry. Dewaxed sections were immersed in 5\% hydrogen peroxide dissolved in absolute methanol for $30 \mathrm{~min}$ utes to quench endogenous peroxidase activity. If antigen retrieval was desirable, as was the case for dysbindin-1, $\beta$-dystrobrevin, and NeuN, the sections were then boiled in $1 \mathrm{mM}$ EDTA in $0.1 \mathrm{M}$ Tris buffer ( $\mathrm{pH}$ 8.0) for 10 minutes (44). After cooling for 20 minutes and rinsing in water, sections later given silver intensification (dysbindin-1, $\beta$-dystrobrevin, and synapsin- 1 ) were soaked in $0.5 \%$ sodium borohydride for 5 minutes. Following two changes of TrisTriton (0.01\% Triton X-100 in 0.1 M Tris-HCl buffer, $\mathrm{pH} 7.6)$, sections processed for all antigens were blocked for 45 minutes in $2 \%$ horse serum dissolved in Tris-Triton and were then incubated in the primary antibody overnight (14-18 hours) at $4^{\circ} \mathrm{C}$. Following Tris-Triton rinses, sections were incubated in a biotinylated secondary antibody (Vector Laboratories Inc., Burlingame, California, USA) for an hour at room temperature, treated for another hour at room temperature with an avidin-biotin-peroxidase complex made

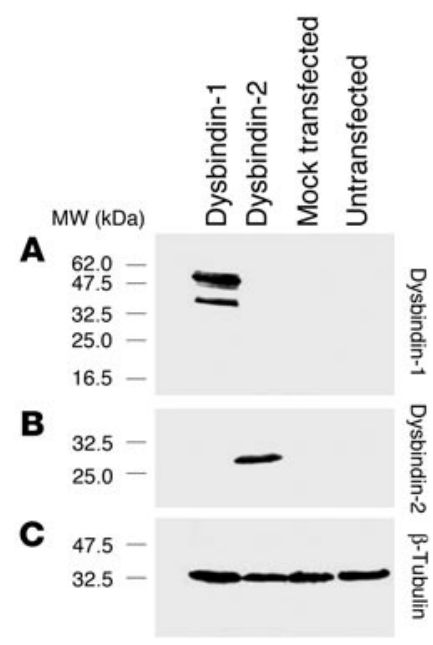




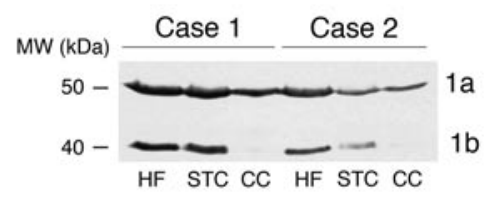

Figure 3

Dysbindin-1 isoforms in human brain regions. PA3111Ae2 (1:100) recognizes two dysbindin-1 isoforms, as shown in a Western blot on two cases here. Lanes were loaded with equal amounts of protein. The cerebellar cortex lacks detectable amounts of isoform $1 \mathrm{~b}$ in both cases. STC, superior temporal cortex; CC, cerebellar cortex.

from a Vectastain Elite ABC kit (Vector Laboratories Inc.), and developed for 10 minutes in a solution containing $0.05 \%$ diaminobenzidine (DAB, HK 124-7K; BioGenex, San Ramon, California, USA ) and $0.03 \%$ hydrogen peroxide (HK 126-7K, BioGenex) in Tris-Triton. For NeuN (1:100), synaptophysin $(1: 1,000)$, and VGluT-1 $(1: 5,000-1: 10,000)$, the DAB solution was supplemented with $0.25 \% \mathrm{NiSO}_{4} .6 \mathrm{H}_{2} \mathrm{O}$ to amplify the immunohistochemical signal. For dysbindin-1 (1:300), $\beta$-dystrobrevin (1:100), and synapsin-1 (1:500), no $\mathrm{NiSO}_{4}$ was added to the DAB solution. Signal amplification for those antigens was maximized instead by on-the-slide silver intensification at $60^{\circ} \mathrm{C}$ for 90 minutes using the method of Teclemariam-Mesbah et al. (45). After clearing in xylenes, tissue sections were coverslipped under Cytoseal 60 (Richard-Allan Scientific, Kalamazoo, Michigan, USA). Dysbindin-1 (1:400) and VGluT-1 $(1: 5,000)$ were colocalized with immunofluorescence using Alexa 594 and 488 secondary antibodies (Molecular Probes Inc., Eugene, Oregon, USA), respectively.

Mouse controls. Male C3H mice $(n=16)$ (Charles River Laboratories Inc., Wilmington, Massachusetts, USA) were used in accordance with NIH guidelines on animal care. Under anesthesia (100 $\mathrm{mg} / \mathrm{kg}$ ketamine and $10 \mathrm{mg} / \mathrm{kg}$ xylazine intraperitoneally), pellets of Medisorb bioabsorbable polymer (Alkermes Inc., Cincinnati, Ohio, USA) were implanted subcutaneously (46). The pellets contained polymer alone in eight of the mice and polymer plus haloperidol (H1512; Sigma-Aldrich, St. Louis, Missouri, USA) in eight other mice. The drug-laden pellets release approximately $2 \mathrm{mg} / \mathrm{kg}$ of haloperidol (Haldol) per day. Ten weeks after pellet implantation, the animals were killed according to a protocol

\section{Figure 4}

Dysbindin-1 immunohistochemistry of the human HF. (A) Diagram of the HF, showing subdivisions and layers. The DGiml is highlighted in red to indicate a major neuropil locus of dysbindin-1. The subjacent granule cell layer is shown in green. (B) Dysbindin-1 immunoreactivity (ir) with PA3111Ae2 (1:300) in the section diagrammed in A. (C and D) Antibody specificity test showing that dysbindin-1 immunoreactivity (C) can be eliminated by preadsorption of $\mathrm{PA} 3111 \mathrm{Ae} 2$ with $10^{-7} \mathrm{M}$ recombinant dysbindin-1, M10FL (D). (E and F) Dysbindin-1-positive neurons in CA3 (E) and the DGh $(\mathbf{F})$. ( $\mathbf{G}$ and $\mathbf{H})$ Dysbindin-1 in the DG. The higher-magnification view in $\mathbf{H}$ shows dendrites of hilar neurons containing dysbindin penetrating the granule cell layer (yellow arrowheads) and extending into the molecular layer (green arrowheads). Note the dense band of presynaptic dysbindin-1 in DGiml. CA3p, stratum pyramidal of CA3; PrS, presubiculum; S, subiculum. Hippocampal layers: I, stratum lucidum; $\mathrm{m}$, stratum moleculare; $o$, stratum oriens; $p$, stratum pyramidal; $r$, stratum radiatum. Components of the DG: g, granule cell layer; $h$, hilus; iml, inner molecular layer; oml, outer molecular layer. Scale bars in A and B: $2 \mathrm{~mm}$; in $\mathbf{C}$ and $\mathbf{D}: 200 \mu \mathrm{m}$; in $\mathbf{E}-\mathbf{H}: 50 \mu \mathrm{m}$. approved by an Institutional Animal Care and Use Committee at the University of Pennsylvania. Their brains were extracted, immersed overnight in neutral-buffered formalin or $70 \%$ ethanol in saline, and then embedded in paraffin blocks for sectioning at $6 \mu \mathrm{m}$. Sections through the HF were reacted for dysbindin-1, $\beta$-dystrobrevin, synapsin-1, synaptophysin, or VGluT-1 as described above for human material. A correction factor for fixation effects on immunoreactivity was used to compare results from mice fixed in formalin versus ethanol.

Image analysis. Immunoreactivity was quantified by net OD, defined as the OD of a region of interest minus the OD of the background (i.e., neighboring white matter). OD analysis was performed on high-resolution, gray-scale photomontages made on a motorized microscope stage with Image-Pro Plus software (Media Cybernetics Inc., Silver Springs, Maryland, USA). Quantitative comparisons of antigens in controls and psychiatric cases were performed only on sections reacted in the same immunohistochemical run and photographed at the same verified light intensity. In addition to OD, Image-Pro Plus was used to quantify neuronal cell areas, neuronal densities, neuropil areas, neuropil densities, and mean laminar thickness. Operators were blind to any identifying information throughout data accrual and analysis.

Statistics. Statistical significance of differences across matched pairs was assessed with the Wilcoxon matched-pairs signed-ranks test. Correlations are given as Spearman $r$ correlation coefficients. $P$ values less than 0.05 were defined as statistically significant.
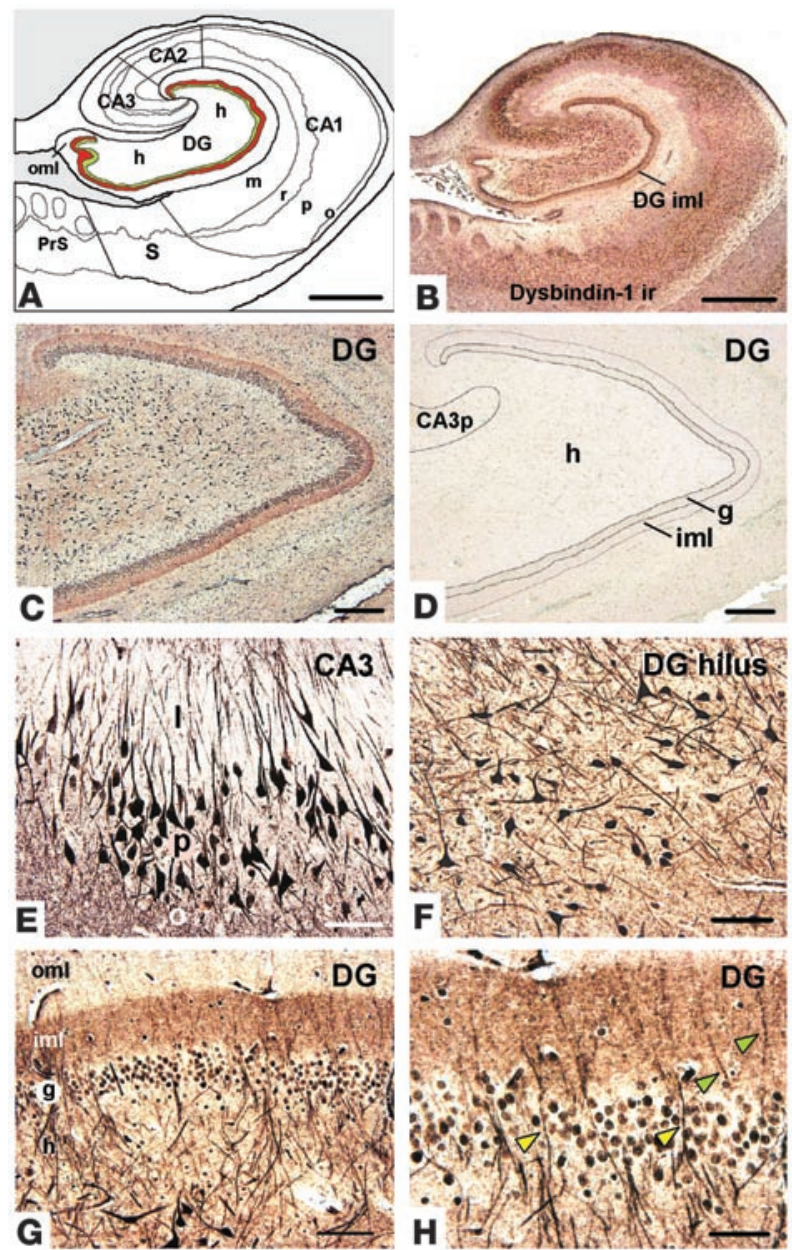

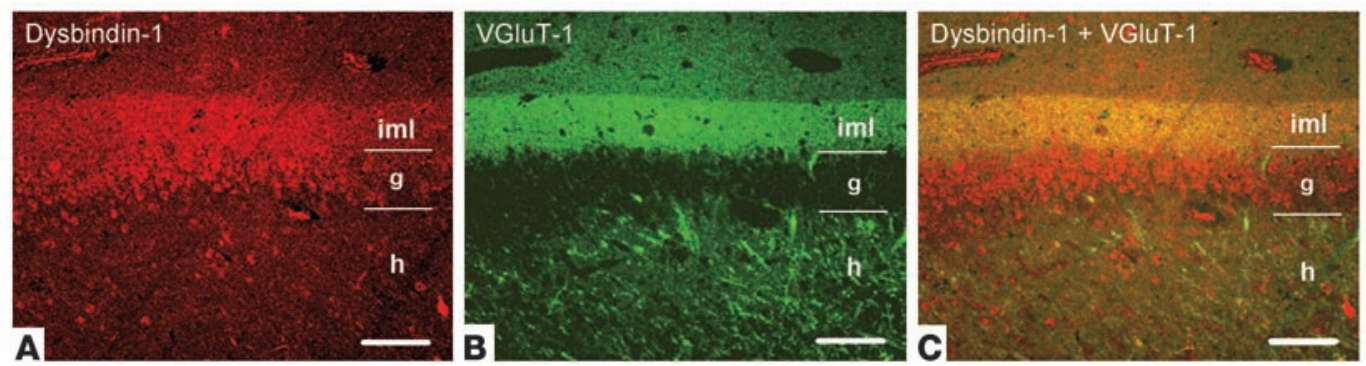

\section{Figure 5}

Colocalization of dysbindin-1 labeled with Alexa 594 (red) and VGluT-1 labeled with Alexa 488 (green) in human DG. The single-channel images in $\mathbf{A}$ and $\mathbf{B}$ are merged in $\mathbf{C}$, which shows that dysbindin-1 and VGluT-1 are coextensive in DGiml. In DGg, only dysbindin-1 is present. In DGh, dysbindin-1 is present in many neurons (not seen clearly here), while VGluT-1 alone is present in mossy fiber terminals of granule cell axons. For abbreviations, see Figure 4. Scale bars: $100 \mu \mathrm{m}$.

\section{Results}

As defined by Scharfman et al. (47), the HF consists of three structures: (a) the hippocampus proper (i.e., cornu ammonis fields 1-3 [CA1-3]); (b) the dentate gyrus (DG); and (c) the subiculum. Their relative locations are indicated in Figures $1 \mathrm{~A}$ and $4 \mathrm{~A}$. Each component of the HF contains a compact neuronal layer (Figure 1A) consisting of small granule cells in the DG and large pyramidal cells in the hippocampus and subiculum $(48,49)$. Dendrites of the compact cell layer extend into cell-poor, synapse-rich layers (e.g., strata oriens and radiatum in the hippocampus and stratum moleculare in the DG; Figure 4A).

Normal dysbindin-1 gene expression. In situ hybridization with an antisense riboprobe (Figure 1) revealed prominent dysbindin-1 gene expression in all principal neuronal populations of the HF, namely pyramidal neurons in the subiculum and CA1-3, granule cells in the dense cell layer of the DG (DGg), and polymorph cells in the hilus of the DG (DGh). All neurons in those populations expressed dysbindin-1 mRNA, but the expression levels varied from low to moderate in CA1 and DGg and high to maximal in CA2, CA3, and DGh (Figure 1, B-E). Variable gene expression also occurred in neurons of neighboring cerebrocortical areas (entorhinal and inferior temporal cortex), though usually at distinctly lower levels than in CA2, CA3, or DGh. There was no evidence of dysbindin-1 gene expression in cells of glial size or in areas rich in glia, such as white matter. No clear cell labeling was observed in the HF or neighboring cerebral cortex with a sense riboprobe (e.g., Figure 1F).

Normal dysbindin-1 isoforms. Western blots demonstrated the presence of dysbindin-1 protein in the HF, as well as in the superior temporal cortex and cerebellar cortex (Figure 3). Two dysbindin-1 isoforms, $1 \mathrm{a}$ and $1 \mathrm{~b}$, were detected with molecular masses of about 50 and $40 \mathrm{kDa}$, respectively. The HF and cerebral cortex contained both isoforms, but the cerebellar cortex lacked the lower-molecular weight isoform (1b), which is a splice variant of full-length dysbindin-1a (25).

Normal dysbindin-1 distribution. Dysbindin-1 immunohistochemistry (Figure 4) was successfully enhanced by heat-induced epitope retrieval (44) and fine-grain silver intensification (45). The distribution of dysbindin-1 protein matched that of dysbindin-1 gene expression. Immunoreactivity was accordingly limited to neurons, was strong in pyramidal cells of CA2 and CA3, and was especially strong in DGh polymorph cells. Neurons in DGg, CA1, the subiculum, and surrounding cortical areas were less immunoreactive (Figure 4, B and E-H). Nevertheless, all principal HF neurons

\section{Figure 6}

Comparison of control (Ctrl) and schizophrenia $(\mathrm{Sz})$ cases for $\beta$-dystrobrevin (A-D), dysbindin-1 (E-H), and VGluT-1 (I-L) in the HF. The blue-gray color of the VGluT-1 panels reflects $\mathrm{NiSO}_{4}$ intensification of immunohistochemical reaction product. Lower-magnification views indicate extent of HF changes in schizophrenia. Note widespread reductions in dysbindin-1 in F. Higher-magnification views of boxed areas of the DG in $\mathbf{A}, \mathbf{B}, \mathbf{E}, \mathbf{F}, \mathbf{I}$, and $\mathbf{J}$ are shown in $\mathbf{C}, \mathbf{D}, \mathbf{G}, \mathbf{H}, \mathbf{K}$, and $\mathbf{L}$, respectively. Note the absence of $\beta$-dystrobrevin in DGiml. Arrows indicate DGiml changes in schizophrenia: a loss of dysbindin-1 $(\mathbf{H})$ and an increase in VGluT-1 (L). For abbreviations, see Figure 4. Scale bars in low-magnification panels: $1 \mathrm{~mm}$; in high-magnification panels: $50 \mu \mathrm{m}$.
Ctrl
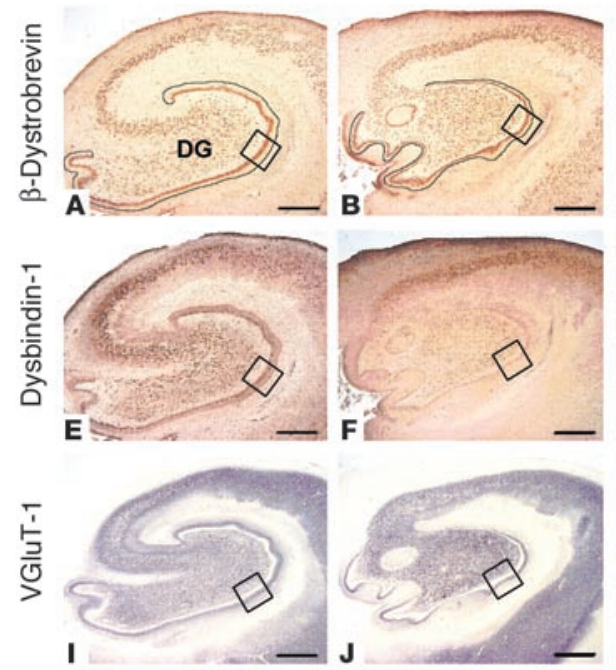

Ctrl

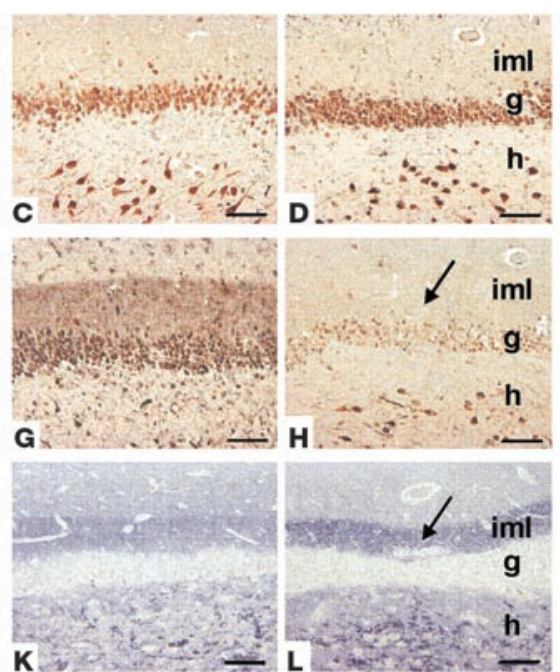




\section{Table 2}

Morphometric comparisons in DG (mean \pm SD)

\section{Feature}

Hilus neuron density (cells $/ \mathrm{mm}^{2}$ ) Hilus dysbindin-1 neuron cell size $\left(\mu \mathrm{m}^{2}\right)$ DGiml depth ( $\mu \mathrm{m}$ in VGluT-1 preparations)
Control cases $(n=17)$

$209.68 \pm 78.31$

$163.57 \pm 26.45$

$70.47 \pm 23.07$
Schizophrenia cases $(n=17)$

$237.28 \pm 75.41$

$164.30 \pm 29.50$

$62.27 \pm 19.55$

\section{Significance of difference}

$P=0.30$

$P=0.94$

$P=0.30$ and their cell nuclei contained dysbindin-1 (Figure 4, E and F). The antigen generally extended far into dendrites only in the strongly immunoreactive neurons of CA2, CA3, and DGh (Figure 4, E and F). Immunoreactivity was occasionally seen there in primary dendritic branches, but never in dendritic spines (Figure 4, E and F).

Dysbindin-1 was not limited to neuronal cell bodies and dendrites, however. The HF was distinguished from most other brain areas examined by bands of diffuse, granular dysbindin immunoreactivity not attributable to somatic, dendritic, or glial labeling. As shown in Figure 4, such immunoreactivity filled three cell-poor, synapse-rich areas: (a) strata oriens and radiatum of CA1-3, (b) the neuropil among pyramidal cells of CA1, and (c) the inner molecular layer of the dentate gyrus (DGiml). Those areas were also filled with diffuse, granular VGluT-1 immunoreactivity (Figures 5B and 6, I and J). Dysbindin-1 thus appeared to be located in axon terminals of glutamatergic pathways arising within (i.e., intrinsic to) the HF (see 49). The protein was not localized in known terminal fields of nonglutamatergic transmitter systems in the HF (i.e., cholinergic, GABAergic, or monoaminergic systems).

Special attention was given to the polymorph neurons in the dentate hilus (Figure 4F). They were richer in dysbindin-1 than any other cells we observed in our survey of the human and the mouse brain. Their density was not significantly different from that of NeuN-labeled neurons in DGh (Table 2 ), indicating that all DGh neurons contain dysbindin-1. Some hilar neurons gave rise to long dysbindin-containing dendrites extending into the inner and outer molecular layers of the DG (Figure 4, $\mathrm{G}$ and $\mathrm{H}$ ). The inner molecular layer (DGiml) was marked by an unusually dense band of diffuse, granular dysbindin-1 likely to be presynaptic as noted above (Figure 4, B, C, G, and H). Colocalization tests showed that the band was coextensive with the glutamate terminal marker VGluT-1 (Figure 5). Those tests also demonstrated that many dysbindin-1 cell bodies in the dentate hilus were encased by VGluT-1 immunoreactivity, presumably in glutamatergic mossy fiber terminals of dentate granule cells.

Normal $\beta$-dystrobrevin distribution. The HF distributions of dysbindin- 1 and $\beta$-dystrobrevin immunoreactivity were discordant. Although both antigens were found in neurons (granule, polymorph, and pyramidal cells; Figure 6, A-H), there was no overlap elsewhere. Of the two antigens, only dysbindin-1 was found in presynaptic fields of the HF (Figure 6; compare A with E and C with G).

Dysbindin-1 in schizophrenia cases. In terminal fields of intrinsic glutamatergic connections of the HF,

Figure 7 dysbindin-1 reductions were common in schizophrenia cases compared with their matched controls. A high percentage of the cases showed such reductions: $75 \%$ in the subiculum, $80 \%$ in CA1, $86 \%$ in CA2/3 stratum oriens, $93 \%$ in CA2/3 stratum radiatum, and $88 \%$ in DGiml (Figures 7 and 8). The mean neuropil reduction in dysbindin-1 immunoreactivity was $18 \%$ in the subiculum (Wilcoxon $W=37.0, P=0.026), 21 \%$ in CA $1(W=38.0, P=0.027), 30 \%$ in $\mathrm{CA} 2 / 3$ stratum oriens $(W=49.0, P=0.003), 37 \%$ in $C A 2 / 3$ stratum radia$\operatorname{tum}(W=51.5, P=0.0001)$, and $42 \%$ in DGiml $(W=71.5, P=0.0003)$ (Figures 7 and 8 ). The neuropil reductions in the subiculum and CA1-3 were not accompanied by significant reductions of dysbindin-1 in the pyramidal cells giving rise to intrinsic, glutamatergic pathways in those HF areas. The parent cells of intrinsic, glutamatergic input to DGiml, however, showed reduced dysbindin- 1 in $65 \%$ of the schizophrenia cases compared with matched controls. The mean loss was $14 \%(W=45.5, P=0.03)$, which was significantly

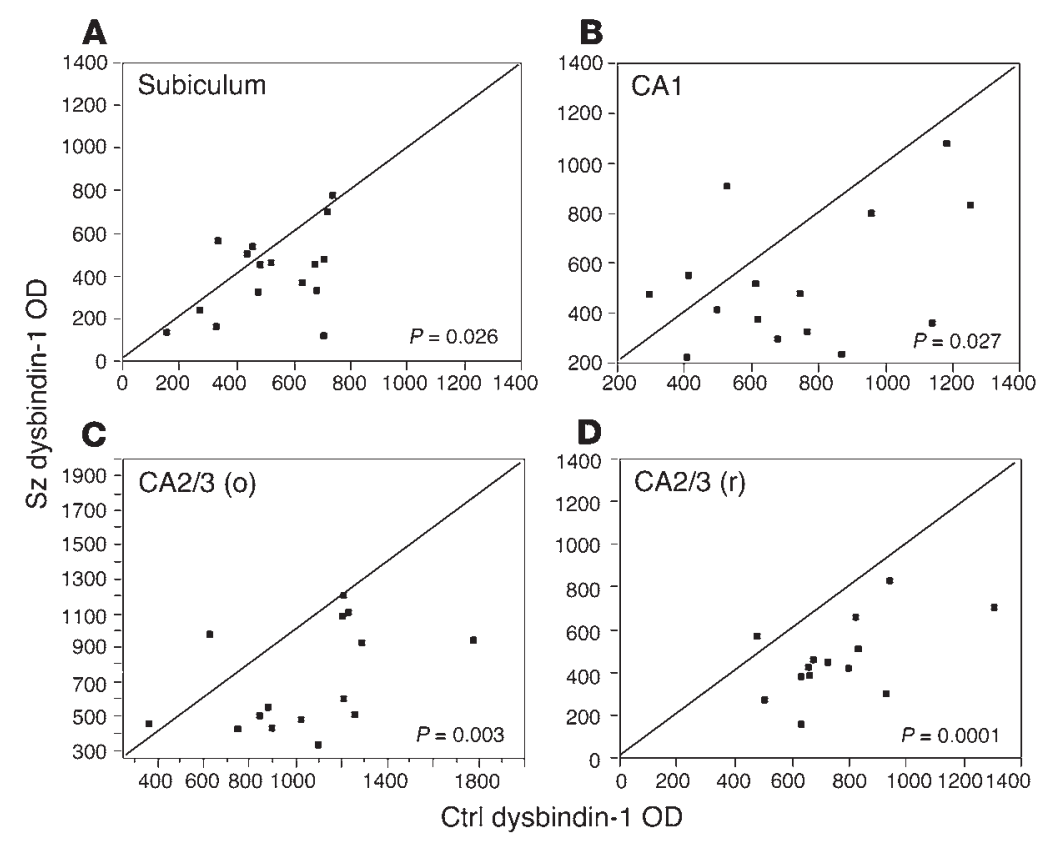

Quantification of dysbindin-1 reductions in extrasomatic (i.e., neuropil) areas of the subiculum and hippocampus proper in schizophrenia compared to matched control cases in the University of Pennsylvania cohort. Data were collected only from neuropil known to contain terminals of glutamatergic pathways arising in the hippocampus. $\mathrm{CA} 2 / 3(\mathrm{o})$ and $\mathrm{CA} 2 / 3(\mathrm{r})$ respectively designate stratum oriens and stratum radiatum of CA2 and CA3. Relative OD was used to quantify dysbindin-1 immunoreactivity. Each data point in these bivariate graphs give the OD of dysbindin-1 immunoreactivity in a schizophrenia case ( $y$ axis) versus that in its matched control ( $x$ axis). Note that $73-93 \%$ of the data points lie below the diagonal lines, indicating that neuropil dysbindin-1 in all four brain areas was lower in the vast majority of the schizophrenia cases compared to matched controls. 
A
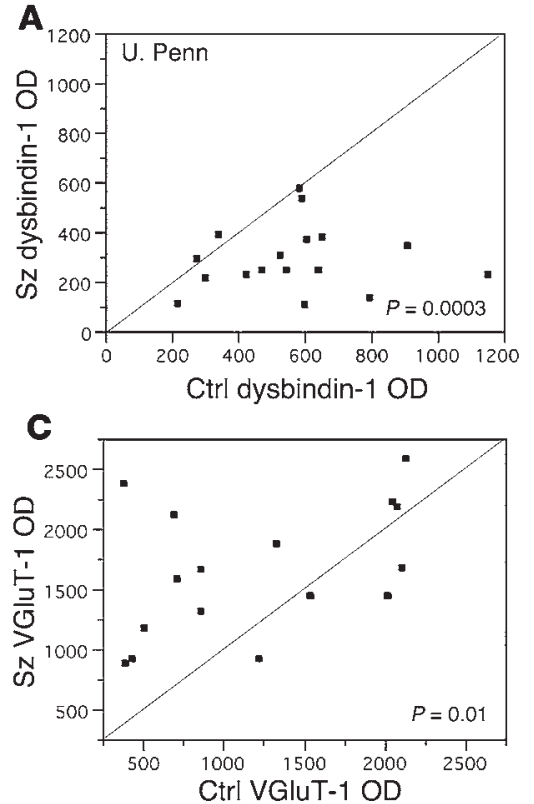

B

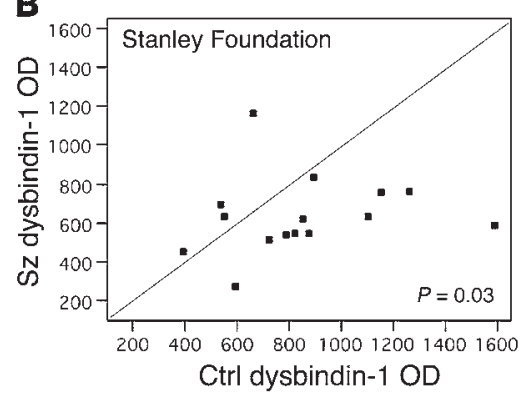

D

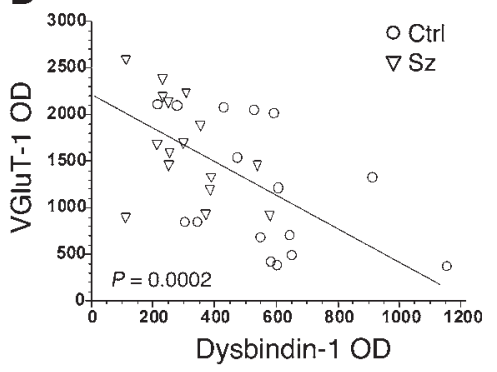

Figure 8

Quantification and correlation of dysbindin-1 and VGluT-1 in DGiml of schizophrenia and matched control cases. (A and B) Bivariate graphs (see Figure 7) showing that schizophrenia cases often display lower dysbindin-1 immunoreactivity in DGiml than matched controls in both the University of Pennsylvania and the Stanley Foundation cohorts. (C) Bivariate graph showing that schizophrenia cases often display higher VGluT-1 immunoreactivity in DGiml than matched controls in the University of Pennsylvania cohort. (D) Correlative levels of dysbindin and VGluT-1 in DGiml of the cases plotted in $\mathbf{A}$ and $\mathbf{C}$. correlated with the mean $42 \%$ loss of dysbindin-1 in DGiml of the same cases (Spearman $r=0.43, P=0.01$ ).

Loss of dysbindin-1 in DGiml was replicated in younger, noninstitutionalized schizophrenia cases from the Stanley Foundation (41). As in the University of Pennsylvania cohort, a high percentage of the cases (73\%) exhibited reduced dysbindin-1 in DGiml compared with matched controls. The mean reduction was $24.9 \%$ ( $W=37.0, P=0.035$; Figure $8 B$ ). Mean reductions of about $18 \%$ were found in DGiml of bipolar and depressive cases from the Stanley Foundation, but they were not significant $(W=20.0$, $P=0.28$ for bipolar cases; $W=31.0, P=0.08$ for depressive cases).

Dysbindin-1 immunoreactivity was nevertheless unaltered in another brain area affected in schizophrenia, specifically the anterior cingulate cortex (31). Analysis of 13 matched pairs from the University of Pennsylvania cohort used for HF analyses revealed no significant changes in cell body or neuropil dysbindin-1 immunoreactivity within superficial or deep layers of the anterior cingulate cortex in schizophrenia ( $W=3.5-13.5, P=0.34-0.84)$. The disorder is thus not characterized by dysbindin-1 abnormalities in all brain areas.

VGluT-1 in schizophrenia cases. As noted above, dysbindin-1 and VGluT-1 are coextensive in DGiml (Figure 5). Since that raises the possibility that the two proteins are colocalized at presynaptic sites, we also measured VGluT-1 immunoreactivity in DGiml. While dysbindin-1 was decreased, VGluT-1 was elevated in $75 \%$ of the schizophrenia cases compared with their matched controls in the University of Pennsylvania cohort (Figures 6, I-L, and 8). The mean VGluT-1 elevation was 84\% ( $W=47.0, P=0.01$; Figure $8 C)$. A significant inverse correlation was found between immunoreactivity for the two proteins in DGiml (Spearman $r=-0.61, P=0.0002$; Figure 8D). Elsewhere in the HF (CA1-3), no significant differences were found in VGluT-1 immunoreactivity between schizophrenia cases and their matched controls.

$\beta$-dystrobrevin in schizophrenia cases. No significant changes in $\beta$-dystrobrevin immunoreactivity were found in the HF of schizophrenia cases compared with matched controls (e.g., Figure 6, A-D).

Analysis of confounding variables. Our matched-pairs design controlled for age, sex, PMI, type of fixative used, and cerebral hemi- sphere studied. Neither dysbindin-1 nor VGluT-1 OD was significantly correlated with subject age, sex, PMI, or antipsychotic exposure measured in equivalent chlorpromazine doses taken daily one month prior to death. Nor did chronic haloperidol treatment of mice significantly affect immunoreactivity for dysbindin-1 (control vs. drug-treated animals [mean \pm SD]: $966.51 \pm 99.52$ vs. $1,054.98 \pm 145.65, t=-1.42, P=0.18)$ or VGluT-1 $(2,016.13 \pm 448.65$ vs. $2,073.65 \pm 159.11, t=1.35, P=0.20)$ in DGiml. The pooled serum level of haloperidol in the drug-treated animals was 3.1 $\mathrm{ng} / \mathrm{ml}$, which is a therapeutic level in humans (50). As detailed in Methods, neither the normal nor the schizophrenia cases exhibited neuropathology. Moreover, the schizophrenia cases showed no loss or shrinkage of DGh neurons (Table 2; consistent with ref. 49), no loss of DGiml thickness (Table 2), and no loss of the synaptic markers synaptophysin or synapsin-1 in DGiml (Figure 9).

\section{Discussion}

We found dysbindin-1 reductions in terminal fields of intrinsic glutamatergic connections in an unusually high percentage of two schizophrenia populations (73-93\% depending on case population and HF area). The average reduction ranged from $18 \%$ in the subiculum to $42 \%$ in DGiml and was significant in both populations studied. No such reductions were observed in bipolar or major-depressive cases, although there was a trend in that direction in the latter cases. Our research design excluded the possibility that the reductions were due to age, sex, neurodegeneration, PMI, fixation, or cerebral hemisphere studied. Neuroleptic medication could also be excluded as a contributing factor. Dysbindin-1 levels in our schizophrenia cases were not correlated with antipsychotic dosage a month before death. Nor did chronic haloperidol treatment of mice affect dysbindin-1 levels in the HF, consistent with the recent finding that haloperidol has no effect on dysbindin-1 levels in the rat brain (22). These considerations suggest that schizophrenia itself is associated with the observed presynaptic dysbindin-1 reductions in the $\mathrm{HF}$.

Possible cause(s) of dysbindin-1 reductions. Reductions in dysbindin-1 gene and protein expression are reported in homogenates of the 

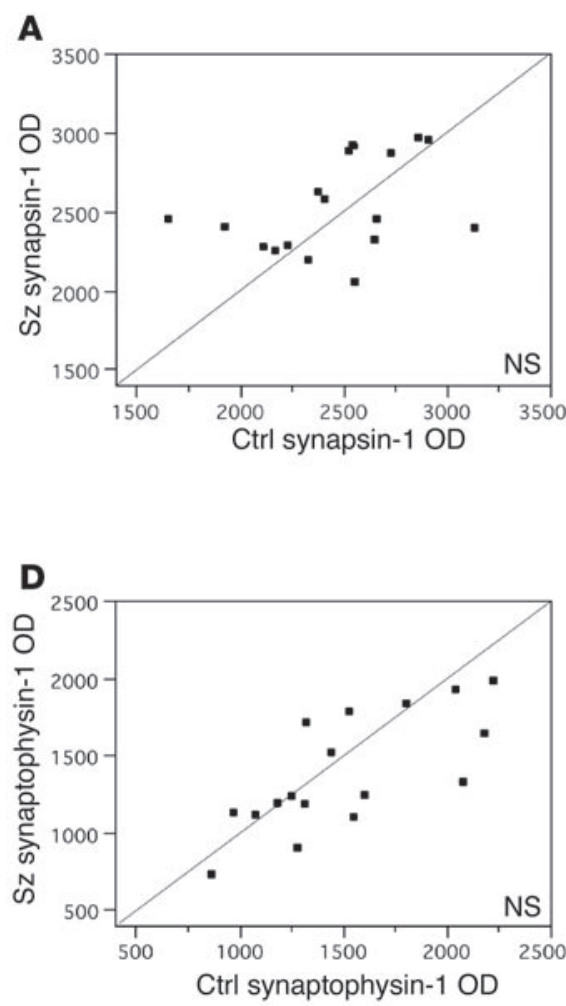

dorsolateral prefrontal cortex in schizophrenia $(23,24)$. Such changes may manifest early in life, given the finding that certain dysbindin-1 polymorphisms are associated with worse premorbid functioning in cases of childhood psychosis (52). It is possible, then, that altered dysbindin-1 expression early in life contributes to schizophrenia onset.

It seems doubtful, however, that dysbindin-1 haplotypes alone account for the protein reductions we observed in schizophrenia cases. The frequency of dysbindin-1 reductions that we observed in schizophrenia cases (73-93\%) was far greater than the frequency of high-risk dysbindin-1 haplotypes $(0-18 \%)$ reported in schizophrenia populations $(10,12,16)$. Other genetic and nongenetic factors would seem to be involved (e.g., genetic epistasis, altered transcriptional regulation, and/or abnormal protein metabolism).

\section{Figure 10}

Summary of results in the context of information flow in the HF. Dysbindin-1 in the HF (A) was found in neurons that supply intrinsic glutamatergic connections in that structure. As diagrammed in B, dysbindin-1 neurons in DGh (red dots) innervate DGiml (red band); those in CA2 and CA3 (green dots) innervate much of CA1-3 (green field); and those in CA1 (yellow dots) innervate the subiculum (yellow field) and entorhinal cortex (ERC). Those intrinsic glutamatergic pathways are part of a broader series of $\mathrm{HFI}$ circuits diagrammed in $\mathbf{C}$. The blue star-bursts there indicate the loci of dysbindin-1 reductions found in schizophrenia, which potentially disrupt normal glutamatergic transmission in the DG, hippocampus proper, and subiculum. See Discussion for consideration of the possible consequences for information flow in the HF. D, dendrites; DGoml, outer molecular layer of the DG; G, dentate granule cell; $\mathrm{H}$, dentate hilar cell; $\mathrm{mf}$, mossy fiber; $\mathrm{pp}$, perforant path; r/o, strata radiatum and oriens; sc, Schaffer collateral. Scale bars in A and B: 2 mm.

\section{Figure 9}

Synapsin-1 and synaptophysin levels in DGiml of schizophrenia matched control cases. No significant differences between schizophrenia and control cases were found in relative OD of either synapsin-1 (A) or synaptophysin (D) immunoreactivity in DGiml. Photomicrographs from sample matched pairs illustrate synapsin-1 (B and $\mathbf{C}$ ) and synaptophysin (E and F) immunohistochemistry in control and schizophrenia cases. Black lines in $\mathbf{E}$ and $\mathbf{F}$ indicate the boundary between the inner and outer molecular layers of the DG, evident in neighboring sections reacted for VGluT-1. The blue-gray color in $\mathbf{E}$ and $\mathbf{F}$ reflects $\mathrm{NiSO}_{4}$ intensification of immunohistochemical reaction product. Scale bars: $100 \mu \mathrm{m}$.

The unusual frequency of the presynaptic protein reductions we observed suggests, moreover, that dysbindin-1 binds unidentified synaptic proteins near the end of a final common pathway by which both genetic and nongenetic factors promote the pathogenesis of schizophrenia.

Synaptic dysbindin-1 and the dystrophin glycoprotein complex. An unexpected conclusion of the present study is that the dystrophin glycoprotein complex is unlikely to be associated with synaptic dysbindin-1 levels in schizophrenia. The synaptic dysbindin-1 reductions we found were presynaptic, whereas the dystrophin glycoprotein complex in brain synapses is exclusively postsynaptic $(28,29)$. The central component of the complex, dystrophin, is found in neurons and dendrites of the human HF but is absent from presynaptic fields of that structure (see ref. 53). We found the same to be true for the dystrophin binding partner $\beta$-dystrobrevin, which was absent from presynaptic fields of the HF. Dysbindin-1 in the HF thus appears to play a presynaptic role independent of $\beta$-dystrobrevin and the dystrophin glycoprotein complex.

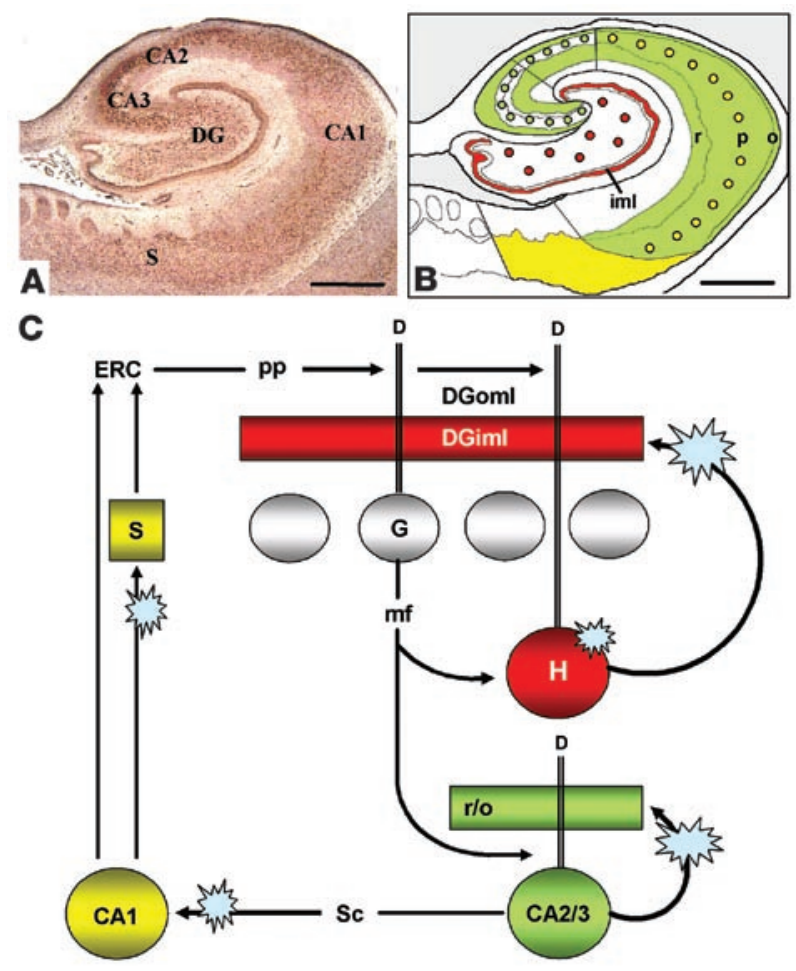


Potential roles of dysbindin-1 in glutamatergic neurotransmission. The presynaptic function of dysbindin-1 may be related to VGluT-1, which is the only vesicular glutamate transporter present in much the HF (43). The inverse relationship we found between the two proteins in DGiml (i.e., a decrease in dysbindin-1 and an increase in VGluT-1; Figure 8D) suggests an effect of dysbindin-1 on VGluT-1 expression, synthesis, or degradation in that HF area. An effect on degradation is consistent with recent evidence that dysbindin-1 binds proteins (muted and pallidin) that promote assembly of lysosome-related organelles (26). Presynaptic lysosomes are, in fact, common in the adult DGiml (54). Elevated VGluT-1 in schizophrenia may thus result from reduced lysosomal activity and/or a compensatory response to known decrements in DG glutamate-receptor expression in the disorder (55-57). Such possibilities deserve further study given mounting evidence for glutamatergic dysfunction in schizophrenia (58) and for an association of that disorder with several genes encoding proteins that affect glutamatergic transmission $(9,59-61)$. An additional possibility is that presynaptic dysbindin-1 reductions can alter glutamatergic transmission without affecting VGluT-1, the levels of which were unaltered in HF areas outside the DGiml in our schizophrenia cases.

Anatomical loci of dysbindin-1 reductions. Dysbindin-1 gene and protein expression were high in the HF compared with surrounding cerebrocortical areas. Both types of expression were highest, however, in the principal neurons of CA2, CA3, and DGh. These cells are the source of intrinsic, glutamatergic connections in the HF (49). Such connections innervate certain cell-poor layers of the hippocampus (i.e., strata oriens and radiatum) and the DG (i.e., its inner molecular layer) (49). These are the same cell-poor layers we found filled with diffuse, granular dysbindin-1 and VGluT-1 immunoreactivity indicative of presynaptic localization. No other synaptic fields in the HF were immunoreactive for dysbindin-1 or VGluT-1.

It is consequently likely that dysbindin-1 neurons of CA2, CA3, and DGh are the source of presynaptic dysbindin-1 within intrinsic, glutamatergic terminals of the HF (Figure 10B). That is especially likely in the case of DGh cells, all of which were found to be intensely immunoreactive for dysbindin-1. DGh cells provide the only substantial input to DGiml (49), which is known to be glutamatergic (62-64). That would explain the significant correlation between the dysbindin-1 reduction in DGh cells and that in DGiml in our schizophrenia cases. We may thus interpret the unusually dense band of presynaptic dysbindin in DGiml as the terminal field of glutamatergic input from DGh, consistent with our finding that dysbindin-1 and VGluT-1 are coextensively distributed in DGiml.

Such observations lead to the conclusion that presynaptic dysbindin in the HF is localized in intrinsic, glutamatergic terminals of that structure. It is in those terminals, then, that reductions in presynaptic HF dysbindin are most likely to occur in schizophrenia. Since the glutamatergic terminals in the areas affected contain VGluT-1 and not VGluT-2 (43), the loss must occur specifically in terminals containing VGluT-1.

Potential consequences of the dysbindin reductions. If dysbindin-1 reduction impairs normal neurotransmission in the DG and CA3 (e.g., via an effect on VGluT-1), information flow through the HF would also be impaired. Figure 10C diagrams the primary course of the information flow, much of which derives from entorhinal cortex input that conveys information from many cerebrocortical areas $(48,49$, $65)$. Via the perforant path, entorhinal input terminates in the outer molecular layer of the dentate gyrus on dendrites of granule (49) and hilar $(66,67)$ cells. As noted earlier, all DGh cells are rich in dysbindin-1 and thus necessarily include DGh cells receiving direct entorhinal input, presumably on dendrites containing dysbindin-1 that we found to extend far into the dentate molecular layer.

DGh cells regulate HF information flow because (a) they have a lower firing threshold for entorhinal input than DGg cells (66, 67), (b) they receive input from DGg cells (49), and (c) they give rise to a recurrent, glutamatergic projection that ends on proximal dendrites of DGg cells over long rostrocaudal segments of the DG (62-64). Via that recurrent projection, DGh cells can prime or recruit large numbers of granule cells to respond to entorhinal input. Moreover, they do so in a manner found to support longterm potentiation in the granule cells (62). As explained above, the recurrent DGh output just described (Figure 10C) terminates in the conspicuous band of dysbindin-1 and VGluT-1 immunoreactivity we observed in DGiml. The dysbindin-1 reduction observed there in schizophrenia cases may thus alter normal information processing and diminish synaptic plasticity in the DG.

Information flow downstream from the DG may also be disrupted by the dysbindin-1 reductions observed in CA3 strata oriens and radiatum of schizophrenia cases. Via mossy fiber projections, DGg cells innervate not only dysbindin-1-positive DGh cells, but also dendrites of dysbindin-1-positive CA3 pyramidal neurons. Those neurons are glutamatergic (68) with many axon collaterals (69), some of which (i.e., Schaffer collaterals) send output further downstream to CA1 and thereby return information back to the entorhinal cortex $(48,49)$. Other collaterals (Figure 10C) are recurrent and innervate cell-poor layers containing dendrites of CA3 pyramidal neurons, including strata oriens and radiatum (69). Such collaterals provide an excitatory-feedback pathway, impairment of which may contribute to the associative memory deficits observed in mice lacking $N$-methyl-D-aspartate (NMDA) receptors specifically in CA3 (70). As in DGh projections, then, reduction of dysbindin-1 in recurrent $\mathrm{CA} 3$ collaterals may alter information processing and diminish synaptic plasticity. The same may be true for CA3 Schaffer collaterals to CA1, excitation of which can normally induce longterm potentiation in CA1 via glutamatergic mechanism (71).

There are thus at least three sites in the HF where presynaptic dysbindin-1 reductions found in schizophrenia may disrupt cognitive processes. The effect would be widespread in the HF, because projections of individual DGh and CA3 pyramidal neurons innervate extensive rostrocaudal segments of the $\operatorname{HF}(49,67)$. Those lengthy association projections are important in forming associations between different sensory modalities, which innervate largely separate rostrocaudal segments of the HF (72). It has been proposed, moreover, that CA3 and DG collectively play an important role in episodic memory, specifically in storage and retrieval of memory sequences in context (73). HF dysbindin-1 losses in schizophrenia may thus contribute to cognitive deficits common in that disorder (74), including those in episodic memory (75).

\section{Acknowledgments}

We express our gratitude to collaborating state hospitals of the Commonwealth of Pennsylvania and, above all, to the patients and their families for their generous participation. We also acknowledge the generous donation of postmortem brain tissue from the Stanley Foundation Brain Collection by the Stanley Medical Research Institute courtesy of M.B. Knable, E. Fuller Torrey, M.J. Webster, S. Weis, and R.H. Yolken. We thank V.M.-Y. Lee and M. Robinson for scientific discussions, W. Bilker for statistical advice, and the clinical staff and residents of the Schizophrenia Center 
and Division of Medical Pathology at the University of Pennsylvania for subject assessment and autopsy. This work was supported by grants from the National Institute of Mental Health (to R.E. Gur and S.E. Arnold), the National Institute on Aging (to J.Q. Trojanowski and S.E. Arnold), and the Wellcome Trust (to D.J. Blake). D.J. Blake is a Wellcome Trust Senior Fellow.

This paper is dedicated to the memory of Denise Talbot (April 23, 1956-October 5, 2002).

1. Owen, M.J., O’Donovan, M.C., and Gottesman, I.I. 2002. Schizophrenia. In Psychiatric genetics and genomics. P. McGuffin, M.J. Owen, and I.I. Gottesman, editors. Oxford University Press. New York, New York, USA. 247-266.

2. Goldner, E.M., Hsu, L., Waraich, P., and Somers, J.M. 2002. Prevalence and incidence studies of schizophrenic disorders: a systematic review of the literature. Can. J. Psychiatry. 47:833-843.

3. Riley, B.P., and McGuffin, P. 2000. Linkage and associated studies of schizophrenia. Am. J. Med. Genet. 97:23-44.

4. Sklar, P. 2002. Linkage analysis in psychiatric disorders: the emerging picture. Annu. Rev. Genomics Hum. Genet. 3:371-413.

5. Lewis, C.M., et al. 2003. Genome scan meta-analysis of schizophrenia and bipolar disorder, part II: schizophrenia. Am. J. Hum. Genet. 73:34-48.

6. O'Donovan, M.C., Williams, N.M., and Owen, M.J. 2003. Recent advances in the genetics of schizophrenia. Hum. Mol. Genet. 12:R125-R133.

7. Owen, M.J., Williams, N.M., and O'Donovan, M.C. 2004. The molecular genetics of schizophrenia: new findings promise new insights. Mol. Psychiatry. 9:14-27.

8. Cloninger, C.R. 2002. The discovery of susceptibility genes for mental disorders. Proc. Natl. Acad. Sci. U.S. A. 99:13365-13367.

9. Harrison, P.J., and Owen, M.J. 2003. Genes for schizophrenia? Recent findings and their pathological implications. Lancet. 361:417-419.

10. Straub, R.E., et al. 2002. Genetic variation in the 6 p22.3 gene DTNBP1, the human ortholog of the mouse dysbindin gene, is associated with schizophrenia. Am. J. Hum. Genet. 71:337-348.

11. Datta, S.R., et al. 2003. Tests of linkage disequilibrium between schizophrenia and genetic markers at the G72 and dysbindin loci. Presentation at: XIth World Congress of Psychiatric Genetics. October 4-8. Quebec City, Quebec, Canada.

12. van den Oord, E.J.C.G., et al. 2003. Identification of a high-risk haplotype for the dystrobrevin binding protein 1 (DTNBP1) gene in the Irish study of high-density schizophrenia families. Mol. Psychiatry. 8:499-510

13. Williams, N.M., et al. 2004. Identification in 2 independent samples of a novel schizophrenia risk haplotype of the dystrobrevin binding protein gene (DTNBP1). Arch. Gen. Psychiatry. 61:336-344.

14. Bakker, S.C., et al. 2003. Association study of the neuregulin, dysbindin and G72 genes in a large sample of Dutch schizophrenic patients. Am. J. Med. Genet. 122B:19. (Abstr.)

15. Schwab, S.G., et al. 2003. Support for association of schizophrenia with genetic variation in the $6 \mathrm{p} 22.3$ gene, dysbindin, in sib-pair families with linkage and in an additional sample of triad families. Am. J. Hum. Genet. 72:185-190.

16. Van Den Bogaert, A., et al. 2003. The DTNBP1 (dysbindin) gene contributes to schizophrenia, depending on family history of the disease. Am.J. Hum. Genet. 73:1438-1443.

17. Kirov, G., et al. 2004. Strong evidence for association between the dystrobrevin binding protein 1 gene (DTNBP1) and schizophrenia in 488 parent-offspring trios from Bulgaria. Biol. Psychiatry. In press.

18. Tang, J.X., et al. 2003. Family-based association
Received for publication October 29, 2003, and accepted in revised form February 3, 2004.

Address correspondence to: Steven E. Arnold, Center for Neurobiology and Behavior, 547B Clinical Research Building, University of Pennsylvania, 415 Curie Boulevard, Philadelphia, Pennsylvania 19104-6140, USA. Phone: (215) 573-2840; Fax: (215) 573-6382; E-mail: sarnold@mail.med.upenn.edu. study of DTNBP1 in 6p22.3 and schizophrenia. Mol. Psychiatry. 8:717-718.

19. Shi, Y.Y., et al. 2003. Non-family based association study of DTNBP1 in 6p22.3 and schizophrenia in geographically and genetically structured Chinese Han population. Am. J. Med. Genet. 122B:102. (Abstr.)

20. Yamada, K., et al. 2003. Suggestive evidence of association between the DTNBP1 gene and schizophrenia in the Japanese samples. Am. J. Med. Genet. 122B: 131

21. Morris, D.W., et al. 2003. No evidence for association of the dysbindin gene (DTNBP1) with schizophrenia in an Irish population-based study. Schizophr. Res. 60:167-172.

22. Maier, W., Zobel, A., and Rietschel, M. 2003. Genetics of schizophrenia and affective disorders. Pharmacopsychiatry. 36(Suppl. 3):5195-5202.

23. McClintock, B.W., et al. 2002. Reduced expression of the common dysbindin transcript in the dorsolateral cortex of schizophrenics. Presentation at: 41st Annual Meeting of the American College of Neuropsychopharmacology. December 8-12. San Juan, Puerto Rico, USA

24. Straub, R.E., et al. 2004. Dysbindin protein is decreased in the dorsolateral prefrontal cortex of schizophrenia patients. Biol. Psychiatry. 55(Suppl. 1):116S. (Abstr.)

25. Benson, M.A., Newey, S.E., Martin-Rendon, E., Hawkes, R., and Blake, D.J. 2001. Dysbindin, a novel coiled-coil-containing protein that interacts with the dystrobrevins in muscle and brain. J. Biol. Chem. 276:24232-24241.

26. Li, W., et al. 2003. Hermansky-Pudlak syndrome type 7 (HPS-7) results from mutant dysbindin, a member of the biogenesis of lysosome-related organelles complex 1 (BLOC-1). Nat. Genet. 35:84-89.

27. Blake, D.J., Weir, A., Newey, S.E., and Davies, K.E. 2002. Function and genetics of dystrophin and dystrophin-related proteins in muscle. Physiol. Rev. 82:291-329.

28. Lidov, H.G.W. 1996. Dystrophin in the nervous system. Brain Pathol. 6:63-77.

29. Blake, D.J., Hawkes, R., Benson, M.A., and Beesley, P.W. 1999. Different dystrophin-like complexes are expressed in neurons and glia. J. Cell Biol. 147:645-657.

30. Blake, D.J., Nawrotzki, R., Loh, N.Y., Górecki, D.C., and Davies, K.E. 1998. $\beta$-Dystrobrevin, a member of the dystrophin-related protein family. Proc. Natl. Acad. Sci. U. S. A. 95:241-246.

31. Benes, F.M. 2000. Emerging principles of altered neural circuitry in schizophrenia. Brain Res. Rev. 31:251-269.

32. Mirnics, K., Middleton, F.A., Lewis, D.A., and Levitt, P. 2001. Analysis of complex disorders with gene expression microarrays: schizophrenia as a disease of the synapse. Trends Neurosci. 24:479-486

33. Bray, N.J., Buckland, P.R., Owen, M.J., and O'Donovan, M.C. 2003. Cis-acting variation in the expression of a high proportion of genes in human brain. Hum. Genet. 113:149-153.

34. Lederhendler, I., and Schulkin, J. 2000. Behavioral neuroscience: challenges for the era of molecular biology. Trends Neurosci. 23:451-454.

35. Knuesel, I., et al. 1999. Altered synaptic clustering of $\mathrm{GABA}_{\mathrm{A}}$ receptors in mice lacking dystrophin
( $m d x$ mice). Eur. J. Neurosci. 11:4457-4462.

36. Brünig, I., Suter, A., Knuesel, I., Lüscher, B., and Fritschy, J.-M. 2002. GABAergic terminals are required for postsynaptic clustering of dystrophin but not of $\mathrm{GABA}_{\mathrm{A}}$ receptors and gephyrin. J. Neurosci. 22:4805-4813

37. Harrison, P.J., and Eastwood, S.L. 2001. Neuropathological studies of synaptic connectivity in the hippocampal formation in schizophrenia. Hippocampus. 11:508-519.

38. Dwork, A.J. 2002. Post mortem studies of the hippocampal formation in schizophrenia. In The postmortem brain in psychiatric research. G. Agam, I.P. Everall, and R.H. Belmaker, editors. Kluwer Academic Publishers. Boston, Massachusetts, USA. 253-266.

39. Schmajuk, N.A. 2001. Hippocampal dysfunction in schizophrenia. Hippocampus. 11:599-613.

40. Arnold, S.E., et al. 1995. Prospective clinicopathologic studies of schizophrenia: accrual and assessment of patients. Am. J. Psychiatry. 152:731-737.

41. Torrey, E.F., Webster, M., Knable, M., Johnston, N., and Yolken, R.H. 2000. The Stanley Foundation brain collection and neuropathology consortium. Schizophr. Res. 44:151-155.

42. Maddox, P.H., and Jenkins, D. 1987. 3-Aminopropyltriethoxysilane (APES): a new advance in section adhesion. J. Clin. Pathol. 40:1256-1260.

43. Kaneko, T., Fujiyama, F., and Hioki, H. 2002. Immunohistochemical localization of candidates for vesicular glutamate transporters in the rat brain. J. Comp. Neurol. 444:39-62.

44. Pileri, S.A., et al. 1997. Antigen retrieval techniques in immunohistochemistry: comparison of different methods. J. Pathol. 183:116-123.

45. Teclemariam-Mesbah, R., Wortel, J., Romijn, H.J., and Buijs, R.M. 1997. A simple silver-gold intensification procedure for double DAB labeling studies in electron microscopy. J. Histochem. Cytochem. 45:619-621.

46. Siegel, S.J., et al. 2002. Surgically implantable longterm antipsychotic delivery systems for the treatment of schizophrenia. Neuropsychopharmacology. 26:817-823.

47. Scharfman, H.E., Witter, M.P., and Schwarcz, R 2000. The parahippocampal region: implications for neurological and psychiatric diseases. Introduction. Ann. N. Y. Acad. Sci. 911:ix-xiii.

48. Insausti, R., and Amaral, D.G. 2004. Hippocampal formation. In The human nervous system. 2 nd edition. G. Paxinos and J. Mai, editors. Academic Press. New York, New York, USA. 871-914.

49. Witter, M.P., and Amaral, D.G. 2004. Hippocampal region. In The rat nervous system. 3rd edition. G. Paxinos, editor. Academic Press. New York, New York, USA.

50. Kapur, S., et al. 1997. The relationship between D2 receptor occupancy and plasma levels on low dose oral haloperidol: a PET study. Psychopharmacology. 131:148-152.

51. Walker, M.A., et al. 2002. Estimated neuronal populations and volumes of the hippocampus and its subfields in schizophrenia. Am. J. Psychiatry. 159:821-828.

52. Gornick, M., et al. 2003. Polymorphisms in dysbindin (DTNBP1, 6p22.3) are associated with intermediate phenotypes measured by the Premor- 
bid Adjustment Scale (PAS) in cases of childhood onset psychosis. 53rd Annual Meeting of the American Society of Human Genetics. November 4-8. Los Angeles, California, USA. (Abstr.)

53. Huard, J., Côté, P.-Y., Parent, A., Bouchard, J.-P., and Tremblay, J.P. 1992. Dystrophin-like immunoreactivity in monkey and human brain areas involved in learning and motor functions. Neurosci. Lett. 141:181-186.

54. Dawirs, R.R., Teuchert-Noodt, G., Hildebrandt, K., and Fei, F. 2000. Granule cell proliferation and axon terminal degradation in the dentate gyrus of gerbils (Meriones unguiculatus) during maturation, adulthood and aging. J. Neural Transm. 107:639-647.

55. Eastwood, S.L., et al. 1995. Decreased expression of mRNAs encoding non-NMDA glutamate receptors GluR1 and GluR2 in medial temporal lobe neurons in schizophrenia. Mol. Brain Res. 29:211-223.

56. Porter, R.H.P., Eastwood, S.L., and Harrison, P.J. 1997. Distribution of kainate receptor subunit mRNAs in human hippocampus, neocortex and cerebellum, and bilateral reduction of hippocampal GluR6 and KA2 transcripts in schizophrenia. Brain Res. 751:217-231.

57. Law, A.J., and Deakin, J.F.W. 2001. Asymmetrical reductions of hippocampal NMDAR1 glutamate receptor mRNA in the psychoses. Neuroreport. 12:2971-2974.

58. Konradi, C., and Heckers, S. 2003. Molecular aspects of glutamate dysregulation: implications for schizophrenia and its treatment. Pharmacol. Ther. 97:153-179.

59. Collier, D.A., and Li, T. 2003. The genetics of schizophrenia: glutamate not dopamine? Eur. J.
Pharmacol. 480:177-184.

60. Moghaddam, B. 2003. Bringing order to the glutamate chaos in schizophrenia. Neuron. 40:881-884.

61. Sawa, A., and Snyder, S.H. 2003. Schizophrenia: neural mechanisms for novel therapies. Mol. Med. 9:3-9.

62. Hetherington, P.A., Austin, K.B., and Shapiro, M.L. 1994. Ipsilateral associational pathway in the dentate gyrus: an excitatory feedback system that supports $\mathrm{N}$-methyl-D-aspartate-dependent long-term potentiation. Hippocampus. 4:422-438.

63. Jackson, M.B., and Scharfman, H.E. 1996. Positive feedback from hilar mossy cells to granule cells in the dentate gyrus revealed by voltage-sensitive dye and microelectrode recording. J. Neurophysiol. 76:601-616.

64. Wenzel, H.J., Buckmaster, P.S., Anderson, N.L., Wenzel, M.E., and Schwartzkroin, P.A. 1997. Ultrastructural localization of neurotransmitter immunoreactivity in mossy cell axons and their synaptic targets in the rat dentate gyrus. Hippocampus. 7:559-570.

65. Witter, M.P., Wouterlood, F.G., Naber, P.A., and van Haeften, T. 2000. Anatomical organization of the parahippocampal-hippocampal network. Ann. N. Y. Acad. Sci. 911:1-24.

66. Scharfman, H.E. 1991. Dentate hilar cells with dendrites in the molecular layer have lower thresholds for synaptic activation by perforant path than granule cells. J. Neurosci. 11:1660-1673.

67. Sik, A., Pentonnen, M., and Buzsáki, G. 1997. Interneurons in the hippocampal dentate gyrus: an in vivo intracellular study. Eur. J. Neurosci. 9:573-588.

68. Vizi, E.S., and Kiss, J.P. 1998. Neurochemistry and pharmacology of the major hippocampal transmitter systems: synaptic and nonsynaptic interactions. Hippocampus. 8:566-607.

69. Ishizuka, N., Weber, J., and Amaral, D.G. 1990. Organization of intrahippocampal projections originating from CA3 pyramidal cells in the rat. J. Comp. Neurol. 295:580-623.

70. Nakazawa, K., et al. 2002. Requirement for hippocampal CA3 NMDA receptors in associative memory recall. Science. 297:211-218.

71. Petrozzino, J.J., and Connor, J.A. 1994. Dendritic $\mathrm{Ca}^{2+}$ accumulation and metabotropic glutamate receptor activation associated with an $\mathrm{N}$-methyl-Daspartate receptor-independent long-term potentiation in hippocampal CA1 neurons. Hippocampus. 4:546-558

72. Small, S.A. 2002. The longitudinal axis of the hippocampal formation: its anatomy, circuitry, and role in cognitive function. Rev. Neurosci. 13:183-194.

73. Lisman, J.E. 1999. Relating hippocampal circuitry to function: recall of memory sequences by reciprocal dentate-CA3 interactions. Neuron. 22:233-242.

74. Gur, R.C., Moelter, S.T., and Ragland, J.D. 2000. Learning and memory in schizophrenia. In Cognition in schizophrenia: impairments, importance, and treatment strategies. T. Sharma and P. Harvey, editors. Oxford University Press. New York, New York, USA. 73-91.

75. Goldberg, T.E., David, A., and Gold, J.M. 2003. Neurocognitive deficits in schizophrenia. In Schizophrenia. 2nd edition. S.R. Hirsch and D.R. Weinberger, editors. Blackwell Publishing. Malden, Massachusetts, USA. 168-184. 